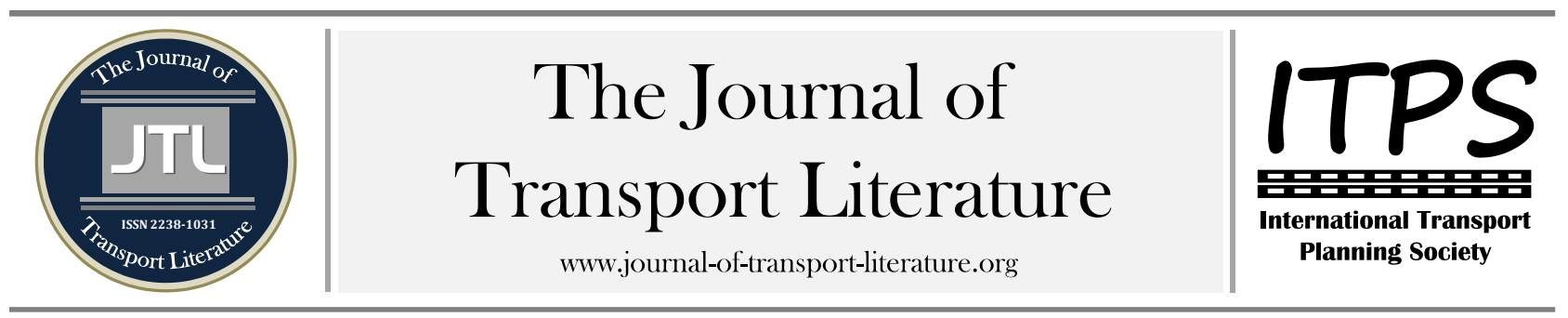

\title{
Modelo de roteirização para a distribuição de materiais de terraplenagem baseado em programação inteira
}

Viviane Adriano Falcão ${ }^{1,+}$; Bruno de Athayde Prata2 ${ }^{2}$ Ernesto Ferreira Nobre Júnior²

${ }^{1}$ Universidade Federal do Triângulo Mineiro, Uberaba,Brasil

${ }^{2}$ Universidade Federal do Ceará, Fortaleza, Brasil

\section{Article Info}

Palavras-chave

Roteirização de Veículos

Equipamentos

Terraplanagem

\section{Resumo}

Alguns estudos afirmam que para obter uma economia geral na construção, os planejadores devem desenvolver uma estratégia de forma a otimizar a utilização dos recursos. Em obras de terraplenagem, uma das formas de fazer isso é minimizar a distância total percorrida pelos veículos na movimentação de terra entre as zonas de corte e aterro. Há muitos estudos que focam na otimização da distribuição de materiais entre zonas de corte e aterro, porém poucos aplicaram em projetos reais, nenhum deles considerou múltiplos equipamentos e nem fizeram uma análise baseada na distância entre estacas. Este trabalho tem como objetivo desenvolver um modelo de Programação Matemática que minimize a distância percorrida pelos caminhões basculantes em atividades de distribuição de materiais na terraplenagem. 0 modelo foi aplicado em um estudo de caso em uma obra rodoviária, no qualse obteve a alocação de corte e aterro ótima, de forma a minimizar a distância percorrida pelos caminhões, e, por conseguinte, a rota ótima para a utilização de caminhões ao longo da obra. Ao comparar o resultado obtido pelo modelo e o resultado fornecido pelo diagrama de massas obtém-se uma economia de $12 \%$ na distância percorrida.
Submitted 1 Jun 2015:

received in revised form 5 Jul 2015

accepted 12 Jul 2015.

Licensed under

Creative Commons

CC-BY $3.0 \mathrm{BR}$

+ Corresponding author. Universidade Federal do Triângulo Mineiro, Instituto de Ciências Tecnológicas e Exatas, Engenharia Civil. Avenida Doutor Randolfo Borges Júnior - 1250. 38064200 - Uberaba, MG - Brasil.

E-mail address: viviane.falcao@uftm.icte.edu.br.

\section{Introdução}

Normalmente, os custos relacionados às obras de terraplanagem equivalem em torno de $25 \%$ do total dos custos em construção de estradas, e isso pode aumentar consideravelmente em função do local onde a estrada será inserida (HARE et al., 2011). Porém Lima (2003) afirma que os custos relacionados a terraplanagem representam a maior parte do custo total das obras rodoviárias.A movimentação de terra, portanto, é um fator significativo na determinação do custo total de um projeto de rodovia. Desta forma, a obtenção do custo mínimo em terraplanagem é essencial para o projeto rodoviário.

Jayawardane e Harris (1990) afirmam que as operações de terraplenagem na construção de rodovias consistem em um importante item da licitação. Logo, a seleção cuidadosa das zonas de empréstimo/bota-fora e dos equipamentos, bem como, a distribuição de materiais é essencial neste processo. Nesse contexto, um modelo computacional que automatiza o processo de minimização de custos em obras de terraplanagem é, portanto, altamente desejável.

El-Rayes e Moselhi (2001) afirmam que otimizar a utilização dos recursos pode levar a uma redução significativa na duração e nos custos dos projetos de construção repetitivos, como rodovias, edifícios e conjuntos habitacionais. Planejar as atividades de distribuição de materiais em obras de terraplanagem pode representar um ganho na obra como um todo.

Askew et al. (2002) afirmam que atividades de terraplenagem consistem tipicamente em escavação (corte), transporte de materiais e depósito de material (aterro). É justamente a seleção e as inter-relações destes cortes e aterros que contribuem para o processo de planejamento da terraplenagem ser complexo. Como há um grande número de combinações dessas atividades em um projeto de rodovias, o planejamento da sua execução precisa ter uma consideração cuidadosa e sistêmica.

Há muitos estudos e trabalhos que aplicam técnicas de otimização matemática em obras de infraestruturas como exemplo: Mayer e Stark (1981), Easa (1987), Christian e Caldera (1988), Easa (1988), Reda (1990), Jayawardane e Harris (1990), Jayawardane e Price (1994a),Huang e Halpin (1995), Henderson et al. (2003), Ipsilandis (2007), Moselhi e Alshibani (2009), Hare et al. (2011) e Nassar e Hosny (2012) porém poucos conseguiram aplicá-los em projetos reais como Nandgaonkar (1981), Jayawardane e Price (1994b), Cristóbal (2009) e Lima et al. (2013), demonstrando que a grande maioria estava mais interessada no aspecto matemático do problema do que na solução da operação de execução.

A Programação Linear é amplamente utilizada na modelagem das operações de terraplanagem devido a sua simplicidade e eficácia. Os trabalhos em sua grande maioria usaram Programação Linear e resolveram a movimentação de terra como sendo um Problema de Transporte. No Problema de Transportes as distâncias entre a origem (corte) e o destino (aterro) são preestabelecidas e as variáveis de decisão são os volumes transportados. Tendo como função objetivo a minimização do custo total de transporte de materiais. 
Nandgaonkar (1981) empregou Programação Linear para resolver o problema do transporte na movimentação de terra para um grande projeto de terraplenagem em Ahmednagar, na Índia. A análise do estudo de caso confirmou que a Programação Linear é uma efetiva ferramenta para encontrar a solução ótima na alocação e, por conseguinte, reduzir os custos na movimentação de terra entre zonas de corte e aterro.

Nassar e Hosny (2012) afirmaram que as formulações típicas do problema movimentação de terra, baseada no Problema de Transportes, não consideram a sequência de movimento dos equipamentos e são, portanto, limitadas na sua capacidade de estabelecer um plano de distribuição de materiais prático e viável.

Limet al. (2005) desenvolveram um modelo usando um algoritmo de recozimento simulado embutido em busca tabu. 0 objetivo era encontrar o menor caminho para o Problema do Caminho Mínimo entre Corte e Aterro. Os autores tentaram estender o modelo para incluir vários veículos, porém a nova formulação não é apresentada. Por conta disso, acredita-se que eles dividiram o problema em dois e somaram as rotas de cada uma das soluções.

Como lacuna na literatura, tem-se a ausência da consideração do uso de múltiplos equipamentos, conforme Henderson et al. (2003) sugerem como estudos futuros para tornar o seu modelo mais realista. Além disso, não é discutida na literatura a questão do uso da distância média de transportes para zonas entre cortes e aterros, situação essa que poderia ser otimizada ao optar-se pela discretização daquelas zonas, através da utilização da distância entre estacas das referidas zonas.

O objetivo deste trabalho é desenvolver um modelo de Programação Linear Inteira que minimize a distância percorrida pelos caminhões basculantes em atividades de distribuição de materiais na terraplenagem. 0 modelo foi aplicado em um estudo de caso na obra da rodovia PE099, onde se obteve a alocação de corte e aterro ótima, de forma a minimizar a distância percorrida pelos caminhões. Por fim, é importa ressaltar que o modelo abordou lacunas da literatura, entre elas o fato de se considerar o problema de roteamento com múltiplos veículos, aplicando-o em um projeto real.

0 trabalho é composto por mais três seções, descritas a seguir. Na próxima, é apresentada uma nova formulação proposta para o problema da movimentação de materiais entre cortes e aterros com o uso de múltiplos caminhões. Na terceira seção é apresentado o estudo de caso, contendo os resultados obtidos, em um projeto real de uma obra de terraplanagem. Por fim, apresentam-se algumas conclusões, e possíveis desenvolvimentos futuros para o trabalho.

\section{Problema de Roteamento entre o Corte e o Aterro com Múltiplos Caminhões}

0 problema de roteamento de corte e aterro é um problema de otimização no qual o objetivo é encontrar uma rota que minimiza a distância total percorrida por uma frota de caminhões utilizada na movimentação de terra. Nesse tipo de problema, as variáveis de decisão são as rotas e as distâncias entre origem (corte) e destino (aterro) são preestabelecidas. E a função objetivo é minimizar a distância total percorrida.

Por se tratar de um modelo baseado no problema de roteamento onde o objetivo é minimizar a distância total percorrida na movimentação de terra entre as zonas de corte e aterro, o número de visitas a uma determinada zona de corte ou aterro vai depender do volume de material necessário para conformá-lo ao greide definido em projeto. 0 modelo trata esse volume como uma unidades de carga equivalente à carga da unidade de transporte a ser utilizada. Uma vez que cada unidade de material representa uma unidade de carga, o número de visitas a cada zona de corte ou aterro é igual à quantidade de excesso (ou déficit) de material no local. Por exemplo, se um determinado corte tiver o volume de $200 \mathrm{~m}^{3}$ para ser retirado e a capacidade do caminhão for de $10 \mathrm{~m}^{3}$, nesse corte haverá 20 unidades de material ou de carga para ser retirada.

0 modelo do problema de roteamento de corte e aterro com múltiplos caminhões é representado conforme equações a seguir:

Índices e conjuntos: $i$ representa o número de cortes, onde $i=1,2, \ldots m ; j$ representa o número de aterros, onde $j=1$, $2, \ldots n ; w$ representa a quantidade de cortes e aterros, ou seja, $m+n ; A$ é o conjunto de arcos de cortes para os aterros e de aterros para cortes; $k$ representa a quantidade de caminhões, onde $k=1,2, \ldots t ; l$ é a localização de cortes e aterros no conjunto $g ; g_{i}$ é o conjunto com a localização dos cortes ou aterros; $L^{+}$é o conjunto das unidades de corte; $L$-é o conjunto das unidades de aterro.

Parâmetros: $p_{i}$ é a posição da unidade de corte ou aterro referente; $D_{i j}$ é a distância média entre o corteie o aterroj;

Variáveis de decisão: $x_{i j k}$ é uma variável binária que assume o valor 1 quando o caminhão $k$ leva o material do corte $i$ para o aterro $j, 0$ caso contrário; $y_{i k}$ é uma variável binária que assume o valor 1 quando o caminhão $k$ leva material do corte $i, 0$ caso contrário;

Função Objetivo:

Sujeito a:

$$
\text { MinimizarZ }=\sum_{(i, j) \in A} D_{i j} x_{i j k}
$$

$$
\begin{gathered}
p_{i}+1-w\left(1-x_{i j k}\right) \leq p_{j} \forall(i, j) \in A, \text { comj } \neq 1, p_{1}=1 \\
x_{i j k}+x_{j i k} \leq 1 \forall(i, j) \in A, \text { comi }<j \\
x_{i j k} \in\{0,1\} \forall(i, j) \in A \\
p_{i} \in\{1, \ldots, l\}, \quad i=1, \ldots, l \\
\sum_{j} x_{i j k}=y_{i k} i=1, \ldots, m \text { e } k=1, \ldots, t
\end{gathered}
$$




$$
\begin{gathered}
\sum_{j} x_{i j k}=y_{j k} j=1, \ldots, n \text { e } \mathrm{k}=1, \ldots, t \\
\sum_{k} y_{i k}=1 i=1, \ldots, n \\
\sum_{i, j} x_{i j k} \leq v k=1, \ldots, t \\
x_{i j k} \in\{0,1\}(i, j) \in A \text { e } k=1, \ldots, t \\
y_{j \mathrm{k}} \in\{0,1\} j=1, \ldots, n \text { e } k=1, \ldots, t
\end{gathered}
$$

A função objetivo (1) prima pela minimização da distância percorrida pelo caminhão que faz movimentação de material entre as zonas de cortes e os aterros. Os conjuntos de restrições representadas pelas equações de (2) e (3) evitam a formação de subcircuitos. Para que os caminhões não parem suas rotas em um corte ou aterro foram inseridos os conjuntos de restrições do tipo (4) e (5), além da restrição (6) que assegura que o caminhão não visite mais de um corte ou aterro de uma vez. 0 conjunto de restrições do tipo (7) equilibra a utilização dos caminhões, sendo que o termo $v$ é um parâmetro que regula a quantidade de viagens realizadas por um dado caminhão. É importante ressaltar que esse balanceamento é flexível, permitindo, portanto, a análise de vários cenários operacionais. Por fim, os conjuntos de restrições dos tipos (8) e (9) dizem respeito ao caráter binário das variáveis de decisão.

\section{Estudo de Caso}

O estudo de caso escolhido foi a rodovia PE099, construída em Pernambuco, na região Nordeste do Brasil, a qual apresenta uma extensão total de $32,12 \mathrm{~km}$. Tendo em vista que em uma obra rodoviária desse porte usualmente é dividida em trechos para sua execução, a PE099 foi dividida em trechos menores, os quais serão otimizados separadamente. Essa divisão não foi simplesmente geométrica, ela foi estabelecida em função da unidade de produção dos equipamentos disponíveis para a execução da obra.

\subsection{Etapas para Resolução do Estudo de Caso}

0 primeiro passo é analisar o trecho em estudo, verificando aspectos como o projeto geométrico e o quadro de cubação, de forma a encontrar o volume de material a ser movimentado. Considerando que normalmente os trechos têm grandes volumes a serem movimentados, se faz necessário dimensionar os equipamentos de tal forma a estabelecer a unidade de produção que melhor se adéqua ao cronograma físico-financeiro da obra. Estabelecido o tipo e a quantidade de equipamentos para executar tal atividade, tem-se, por conseguinte, a unidade de produção horária. De posse da produção horária, encontra-se a produção por semana e em função dessa produção, ou seja, a quantidade de volume a ser movimentada, estabelece-se qual unidade de tempo será considerada. Para o estudo de caso em questão, foi considerada uma produção de $153 \mathrm{~m}^{3} / \mathrm{h}$, o que equivale a um volume de cerca de $6.000 \mathrm{~m}^{3}$ por semana. É importante ressaltar que essa produção horária é diretamente relacionada com os equipamentos, suas características e sua quantidade. Nesse caso, a equipe escolhida foi: 1 carregadeira com $3 \mathrm{~m}^{3}$ de caçamba e 4 caminhões basculantes cada um com $14 \mathrm{~m}^{3}$.

A etapa seguinte é a divisão dos trechos da rodovia de acordo com essa produção.Nesse caso, optou-se por a divisão em trechos de mais ou menos $6.000 \mathrm{~m}^{3}$, visto que seria a produção semanal da equipe dimensionada. Onde o primeiro trecho que foi aplicado o modelo foi o subtrecho TR-1A, em torno de $3 \mathrm{~km}$ de extensão.Considerando que a unidade de carga do modelo é função da capacidade de carga do caminhão basculante, os volumes em cada corte ou aterro foram divididos por $14 \mathrm{~m}^{3}$. Posteriormente, é determinada a matriz de distâncias entre os cortes e aterros, a partir do quadro de cubação, em função do estaqueamento com o auxílio do Excel. Outros dados de entrada exigidos pelo modelo também são estabelecidos, quais sejam: o conjunto da distância entre cada corte e aterro, o conjunto de volumes de cortes e o conjunto de volumes de aterros. Para finalizar os dados são inseridos e rodados no CPLEX versão 12.6, executado em um sistema operacional Windows 8.1 de 64 Bits, com processador Core i5 e 4 GB de memória RAM.

\subsection{Resultados}

Para definir a movimentação de terra, a empresa responsável por essa obra, utilizou o diagrama de massas, que para o trecho em questão tem como solução o momento de transporte de $3.401,58 \mathrm{~m}^{3} . \mathrm{km}$, enquanto que a solução obtida pelo modelo proposto foi de $2.994,88 \mathrm{~m}^{3} . \mathrm{km}$, conforme ilustrado na Tabela 1. Deve-se observar que a solução gerada pelo modelo acarretou em uma diminuição de $12 \%$ da distância percorrida pelos caminhões.

Tabela 1 - Estatísticas dos caminhões.

\begin{tabular}{cccccc}
\hline & Viagens & $\begin{array}{c}\text { Distância } \\
(\mathbf{k m})\end{array}$ & Utilização & $\begin{array}{c}\text { Volume } \\
\text { Movimentado } \\
\left(\mathbf{m}^{\mathbf{3}}\right)\end{array}$ & $\begin{array}{c}\text { Momento de } \\
\text { Transporte } \\
\left(\mathbf{m}^{\mathbf{3}} . \mathbf{k m}\right)\end{array}$ \\
\hline Caminhão 1 & 99 & 56,10 & $26 \%$ & 1386 & 785,40 \\
Caminhão 2 & 99 & 58,80 & $27 \%$ & 1386 & 823,20 \\
Caminhão 3 & 98 & 55,42 & $26 \%$ & 1372 & 775,88 \\
Caminhão 4 & 97 & 43,60 & $\mathbf{2 0 \%}$ & 1358 & 610,40 \\
\hline TOTAL & $\mathbf{3 9 3}$ & $\mathbf{2 1 3 , 9 2}$ & $\mathbf{5 5 0 2}$ & $\mathbf{2 9 9 4 , 8 8}$ \\
\hline \multicolumn{7}{c}{ Fonte: Autores }
\end{tabular}

Outro fator verificado foi a utilização de cada um dos caminhões, conforme ilustrado na Figura 1. Nesse gráfico tem-se a utilização por caminhões em duas situações. A primeira se refere ao modelo com a restrição da inequação (7), que promove o balanceamento da utilização dos caminhões, fixando em um máximo de 99 viagens por caminhão. Percebe-se através da 
Figura 1, que esse balanceamento também é expresso em termos de momento de transporte, e, por conseguinte,da distância percorrida.Conforme ilustrado na Tabela 1, somente o caminhão 4, tem uma utilização abaixo dos $25 \%$.

Ainda com relação à Figura 1, pode-se observar que ao rodar o modelo sem a restrição de balanceamento, percebe-se claramente que há uma sobrecarga de utilização dos caminhões 1 e 2, enquanto os caminhões 3 e 4 estão trabalhando abaixo do esperado.

A função objetivo forneceu uma distância total percorrida pelos caminhões de 213,92 km. Ao comparar esse resultado com o momento de transporte calculado através do diagrama de massas, tem-se uma diminuição de cerca de $12 \%$ da distância percorrida pelos caminhões, comprovando dessa forma a eficácia do modelo acima exposto. Para obtenção da solução ótima, o tempo computacional do CPLEX para subtrecho TR-1A foi de aproximadamente 16 minutos.

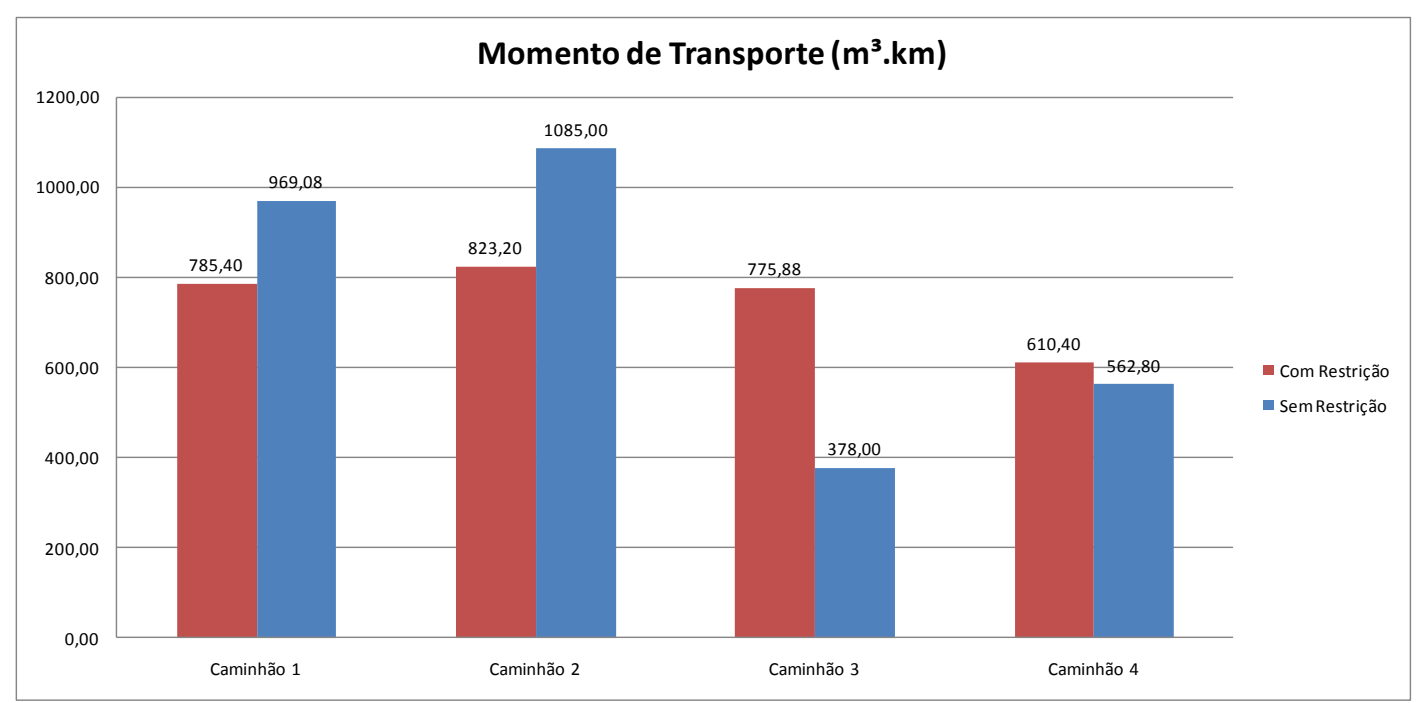

Figura1 - Momento de Transporte por caminhões.

Fonte: Autores

Foi analisado também o resultado proposto pelo modelo para a movimentação de terra entre as zonas de corte e aterro. Na Tabela 2, tem-se um comparativo da distribuição proposta pela construtora e a distribuição proposta pelo modelo. Em termos de volume, percebe-se que não existe diferença significativa entre as duas alocações; contudo, acredita-se que, como foi considerada no modelo a distância média entre as estacas em detrimento da distância média de transportes, tem-se uma análise mais acurada da situação e, por conseguinte, uma redução considerável na distância percorrida e no momento de transporte.

Tabela 2 - Comparativo entre a Movimentação de Terra MOVIMENTAÇÃO DE TERRA

\begin{tabular}{|c|c|c|c|c|c|}
\hline \multicolumn{3}{|c|}{ PRÁTICA } & \multicolumn{3}{|c|}{ MODELO } \\
\hline CORTE & ATERRO & VOLUME $\left(\mathrm{m}^{3}\right)$ & CORTE & ATERRO & VOLUME $\left(\mathrm{m}^{3}\right)$ \\
\hline $\mathrm{C} 1$ & $\mathrm{~A} 1$ & 31 & $\mathrm{C} 1$ & $\mathrm{~A} 1$ & 28 \\
\hline $\mathrm{C} 2$ & A1 & 20 & $\mathrm{C} 2$ & A1 & 28 \\
\hline C3 & $\mathrm{A} 1$ & 41 & $\mathrm{C} 3$ & $\mathrm{~A} 1$ & 56 \\
\hline C3 & A2 & 282 & C3 & A2 & 266 \\
\hline $\mathrm{C} 3$ & A3 & 73 & $\mathrm{C} 3$ & A3 & 84 \\
\hline C3 & A4 & 118 & C3 & A4 & 126 \\
\hline $\mathrm{C} 4$ & A4 & 12 & $\mathrm{C} 4$ & A4 & 14 \\
\hline C5 & A4 & 2.640 & C5 & A4 & 2.618 \\
\hline $\mathrm{C} 5$ & A5 & 741 & C5 & A5 & 728 \\
\hline C5 & A6 & 410 & C5 & A6 & 406 \\
\hline $\mathrm{C} 6$ & \begin{tabular}{l|l}
$\mathrm{A} 6$ \\
\end{tabular} & 1.133 & $\mathrm{C} 6$ & $\mathrm{~A} 6$ & 1.148 \\
\hline \multicolumn{2}{|c|}{ MOMENTO DE TRANSPORTE $(\mathrm{m} 3 . \mathrm{Km})$} & $3.401,58$ & \multicolumn{2}{|c|}{ MOMENTO DE TRANSPORTE (m3.Km) } & $2.994,88$ \\
\hline
\end{tabular}

\section{Conclusões}

Os diferenciais do modelo abordado no trabalho em questão consistem no fato da modelagem do problema não ser através problema de transporte, como ocorre com praticamente todos os trabalhos apresentados na literatura de revisão, bem como o fato de que nenhum dos trabalhos anteriores abordou o problema com múltiplos equipamentos. Além disso, ele se torna um dos poucos trabalhos que utilizaram técnicas de Programação Linear e que conseguiram aplicar os respectivos modelos em um estudo de caso real.

Além da redução no momento de transporte, provavelmente ocasionada pelo fato que o modelo consegue fazer uma análise mais apurada das distâncias reais entre as zonas de corte e aterro, tem-se a alocação ótima da distribuição de 
materiais. Outro ponto positivo do modelo é a opção de promover o balanceamento na utilização dos caminhões basculantes auxiliando dessa forma o uso racional dos mesmos.

Para estudos futuros, propõe-se a extensão desse modelo para um modelo que considere equipamentos heterogêneos. Outra sugestão para estudos futuros seria a automatização da obtenção dos dados de entrada e saída do modelo, tanto no aspecto de banco de dados quanto na impressão dos resultados.

Automatizar a geração das soluções no processo de planejamento minimiza o tempo e o esforço despendido, se comparado ao planejamento realizado manualmente. Isso pode ser comprovado através do tempo que foi necessário ao modelo para obter a solução ótima para o subtrecho TR-1A, não mais do que 16 minutos para encontrar a solução ótima.

\title{
Agradecimentos
}

A primeira autora agradece à CAPES pela bolsa de estudos. 0 terceiro autor agradece ao CNPq pelo apoio financeiro fornecido (Edital MCT/CNPq 14/2012 Processo 470609/2012-5).

\section{Referências}

Askew, W.H. , Al-jibouri, S.H.,Mawdesley, M.J. and Patterson, D.E. (2002). Planning linear construction projects: automated method for the generation of earthwork activities. Automation in Construction, 11, 643-653

Christian, J., and Caldera, H. (1988). Earthmoving cost optimization by operational research. Canadian Journal of Civil Engineering, 15, 679684.

Cristóbal, J. R. S. (2009). Time, Cost, and Quality in a Road Building Project.Journal of Construction Engineering and Manage ment.135, 12711274.

Henderson,D., Vaughan, D.E.,Jacobson, S.H.,Wakefieldd,R. R.andSewell, E. C. (2003). Solving the shortest route cut and fill problem using simulated annealing/ European Journal of Operational Research, 145,72-84

Easa, S. (1987). Earthwork Allocations With Nonconstant Unit Costs. Journal of Construction Engineering and Management, 113,34-50.

Easa, S. (1988).Earthwork Allocations with Linear Unit Costs. Journal of Construction Engineering and Management, $114(4), 641-655$.

El-Rayes, K. and Moselhi, O. (2001).Optimizing Resource Utilization for Repetitive Construction Projects. Journal of Construction Engineering and Management, 127(1), 18-27.

Hare, L. W., Koch, V. R. andLucet, Y. (2011). Models and algorithms to improve earthwork operations in road design using mixed integer linear programming. European Journal of Operational Research, 215, 470-480.

Huang, R and Halpin, D. (1995). POLO: Planning and Optmization for Linear Operations. Proceedings of the 1995 Second Congress on Computing in Civil Engineering, Atlanta, GA, 1, 1126-1133.

Ipsilandis, P. G. (2007). Multiobjective Linear Programming Model for Scheduling Linear Repetitive Projects.Journal of Construction Engineering and Management, 133, 417-424.

Jayawardane, A. K. W., \& Price, A. D. F. (1994a). A New Approach for Optimizing Earth Moving Operations, Part I, In: Proceedings of Institution of Civil Engineers - Transport, 105, 195-207.

Jayawardane, A. K. W., \& Price, A. D. F. (1994b). A New Approach for Optimizing Earth Moving Operations, Part II, In: Proceedings of Institution of Civil Engineers - Transport, 105, 249-258.

Jayawardane, A. K. W., and Harris, F. C. (1990) Further development of integer programming in earthwork optimization, Journal of Construction Engineering and Management, 116, 18-34

Lim, A, Rodrigues, B and Zhang, J. (2005). Tabu search embedded simulated annealing for the shortest route cut and fill problem. Journal of the Operational Research Society, 56, 816-824.

Lima, R. X. (2003). Logística da Distribuição de Materiais em Pavimentação Rodoviária - Uma Modelagem em Programação Matemática. Dissertação de Mestrado - Programa de Mestrado em Engenharia de Transportes, Centro de Tecnologia, Universidade Federal do Ceará, Fortaleza, CE, $288 \mathrm{fl}$.

Lima, R. X. de; Nobre Júnior, E. F.; Prata, B. de A. and Weissmann, J. (2013). Distribution of materials in road earthmoving and paving: a mathematical programming approach. Journal of Construction Engineering and Management, 139, 1046-1054.

Mayer, R. H. and Stark, R. M. (1981), Earthmoving Logistics. Journal of the Construction Division, 107(2), 297-312

Moselhi, O. and Alshibani, A. (2009).Optimization of Earthmoving Operations in Heavy Civil Engineering Projects. Journal of Construction Engineering and Management,135, 948-954.

Nandgaonkar, S. M. (1981), Earthwork Transportation Allocations: Operations Research. Journal of ConstructionDivision, ASCE,107, (2),373-392.

Nassar, K. and Hosny, O. (2012).Solving the Least-Cost Route Cut and Fill Sequencing Problem Using Particle Swarm. Journal of Construction Engineering and Management, 138(8), 931-942.

Reda, R. (1990). RPM: Repetitive Project Modeling. Journal of Construction Engineering and Management.116(2), 316-330.

\begin{abstract}
Some studies claim that for an overall savings in the construction, planners should develop a strategy to optimize resource utilization. In Earthworks one way to do this is to minimize the total distance traveled by trucks between the cut and fill areas in earthworks. There are many studies and papers that focus on the optimization of distribution of material between cut and fill areas, but few applied in real projects, none of them considered multiple equipment and neither did an analysis based on the distance between piles. This study aims to develop a mathematical programming model that minimizes the distance traveled by trucks in materials distribution of earthmoving activities. It was applied in case study the road works, which obtained allocating optimal to cut and fill areas in order to minimize the distance traveled by the trucks, and consequently, the optimum route to trucks. Comparing the model's result with the mass diagram it obtained a saving of $12 \%$ the distance.
\end{abstract}

Key words: Vehicle Routing Problem, equipments, earthworks. 